\title{
CALIBRATION OF A SPATIAL SIMULATION MODEL WITH VOLUNTEERED GEOGRAPHICAL INFORMATION
}

\begin{abstract}
For many scientific disciplines, the continued progression of information technology has increased the availability of data, computation, and analytical methodologies including simulation and visualisation. Geographical Information Science is no exception. In this paper, we investigate the possibilities for deployment of e-infrastructures to inform spatial planning, analysis and policy-making. We describe an existing architecture which feeds both static and dynamic simulation models from a variety of sources, including not only administrative data sets but also attitudes and behaviours which are harvested online from crowds. This infrastructure also supports visualisation and computationally intensive processing.

The main aim of the paper is to illustrate how spatial simulation models can be calibrated with crowd-sourced data. We introduce an example in which popular attitudes to congestion charging in a major UK city (Manchester) were collected, with promotional support from a high profile media organisation (the $\mathrm{BBC}$ ). These data are used to estimate the parameters of a transport simulation model, using a hungry estimation procedure which is deployed within a high performance computational grid. We indicate how the resulting model might be used to evaluate the impact of alternative policy options for regulating the traffic in Manchester.

Whilst the procedure is novel in itself, we argue that greater credibility could be added by the incorporation of open source simulation models, and by the use of social networking mechanisms to share policy evaluations much more widely.
\end{abstract}

\section{Keywords}

Crowd-sourcing; simulation; calibration; e-infrastructure. 


\section{Introduction}

Scientific research is being fundamentally transformed by continued progression in computational technologies. The ability to share information across the internet has now been supplemented by the development of web services permitting complex transformation of increasingly massive datasets. In many disciplines, it is now argued that data intensive research is leading on to a new paradigm of scientific investigation (Bell, Hey, and Szalay, 2009).

These trends are obviously relevant in the domains of GIS and spatial science. Of particular importance is the increasingly widespread availability of spatial data. Thanks to initiatives as diverse as Google Earth and OpenStreetMap, for example, new map-based representations of the physical environment are being created and updated. Existing providers, such as Ordnance Survey in the UK, are becoming obliged to make data more widely available through a combination of commercial and regulatory pressures. A second important dynamic is growing interest in model-based applications, with a special emphasis on multi-agent systems - an overview is provided by Epstein (2007) and notable examples with a geographical emphasis include UrbanSim (Waddell and Borning, 2002), ILUTE (Miller et al, 2004) and Episims (Eubank et al, 2004) amongst many others. The convergence of data manipulation, visualisation and spatial analysis and modelling gives rise to an emergent sub-discipline of geosimulation (Benenson and Torrens, 2004).

An e-infrastructure for social and spatial simulation which brings together these fundamental components of data integration, analytic simulation and visualisation is reviewed in Section 2 of the paper. The rationale for this project combines both an academic and a more applied policy dimension. In the remainder of the paper, the applied value of this work is demonstrated in the context of a real planning scenario, in which the impact of a transport policy initiative on a UK city is evaluated. In the third section of the paper, the transport simulation component of the infrastructure is described, before we go on to discuss the way in which individual behavioural data is captured and incorporated within the model calibration process. The resulting models are deployed in a policy analysis context. The paper ends with a discussion of results and a statement of the major implications for the immediate research agenda.

This work is distinctive in seeking explicitly to drive a simulation from 'crowd-sourced' data which captures the attitudes and stated behaviours of a sub-population and to use this data to enhance the specification and calibration of the model. A high performance infrastructure is used as the means for detailed parameterisation of the model outputs.

\section{The Genesis Infrastructure}

In this section, we describe briefly the two major components which are combined as the core of our approach - a series of demographic models ('Moses') which are used as the basis for small area representation and projection of local populations, and visualisation functionality ('MapTube') which provides a flexible mechanism for the representation of model data, 
analytics and simulation results. In the third part of the section, we review the architecture by which these components are brought together.

\section{$2.1 \quad$ Moses demographic models}

Moses is a microsimulation framework comprising a synthetic estimation procedure or Population Reconstruction Model (PRM) and a dynamic simulation model (DSM). The PRM allows the population of a geographical area to be represented as a sequence of individual households and their constituent members. In addition to socio-demographics, behaviours, attitudes and micro-locations can all be captured from the combination of anonymised individual microdata and neighbourhood level census-based population counts (Birkin et al, 2009). Although the population reconstruction process is relatively complicated, an important advantage is that individually specified household units provide the perfect basis for the simulation of population dynamics. The DSM simulates fundamental demographic transitions using evidence from a variety of longitudinal and dynamic sources, including British Household Panel Survey, International Passenger Statistics, Special Migration Statistics, and Vital Statistics from the Office for National Statistics. The model has been enhanced for specific applications through the introduction of agent-based representation of demographic interactions (Wu et al, 2008, 2010). In summary, Moses provides synthetic individual datasets for both existing and future populations with the capacity for flexible disaggregation across a combination of sociodemographic, attitudinal and micro-locational characteristics.

\subsection{MapTube visualisations}

The MapTube website has been originally conceived as a repository or 'a place to put maps' and was designed for disseminating geographic information to the general public (http://www.maptube.org). Subsequently, the technology has been extended through the GMAP Creator product which allows users to combine attributes for small geographical areas with boundary data which specifies the extent of each area, and underlying representations of the background geography, for example as Google Maps. Furthermore, the technology has been extended to allow maps to be created dynamically by combining attribute data with boundary data to create thematic overlays on Google Maps or OpenStreetMap. The latest innovation in the MapTube portfolio is SurveyMapper (http://www.surveymapper.com) which allows registered users to easily generate and distribute survey questionnaires to a wide variety of potential respondents. SurveyMapper allows the input of user generated content into the simulation process. There is no widely accepted, precise definition of user-generated content, but it is generally considered to be content that: is made publically available over the Internet; reflects a degree of creative effort; and is created outside of professional routines and practices (OECD, 2007). With respect to mapping there are two aspects that are important: crowdsourcing location-related data to create maps and crowdsourcing other data to overlay onto a map backcloth (Anand et al. 2010). When user-generated or crowd sourced data is applied to a mapping context it can be viewed as Volunteered Geographic Information, (Goodchild, 2007). In our case SurveyMapper is a public data collection tool with elements of both crowd sourcing and Volunteered Geographic Information. In Section 3 below we will explain how SurveyMapper has been used proactively to generate crowd-sourced survey data.

\section{3 e-infrastructure for social and spatial simulation}


In recent years, GIS technologies have developed to combine spatial data with analytical functions, and to visualise the resulting outcomes. Specific large-scale projects often combining data with customised spatial analysis or modelling capabilities can give rise to spatial decision support systems for planning and policy-making (for further discussion and a range of examples, see Geertman and Stillwell, 2003). In this research, we seek to combine a similar range of components within the framework of an e-infrastructure, which we see as applicable to a range of problem domains in both the spatial and social sciences. The breadth and ambition of the project are expressed in its characterisation as a National e-Infrastructure for Social Simulation (NeISS: Birkin et al, 2010).

The overall architecture for NeISS is illustrated in Figure 1. The data sources which are enabled by the e-infrastructure include both established geospatial and social data sources such as the Census of Population and Households, government surveys and map data, as well as crowd sourced data which is unique to this project and which we will discuss in more detail at Section 3.1 below. These data feed into the demographic models - PRM and DSM - as we have already described above, as well as a suite of simulation models which we will illustrate further below. For other social modelling applications a data fusion tool is under development in which, for example, the relationships between social background, employment and education can be explored in a variety of information environments and policy contexts (Lambert et al, 2010). The MapTube component allows for the spatial representation and visualisation of model outputs.

Figure 1. The NeISS Architecture

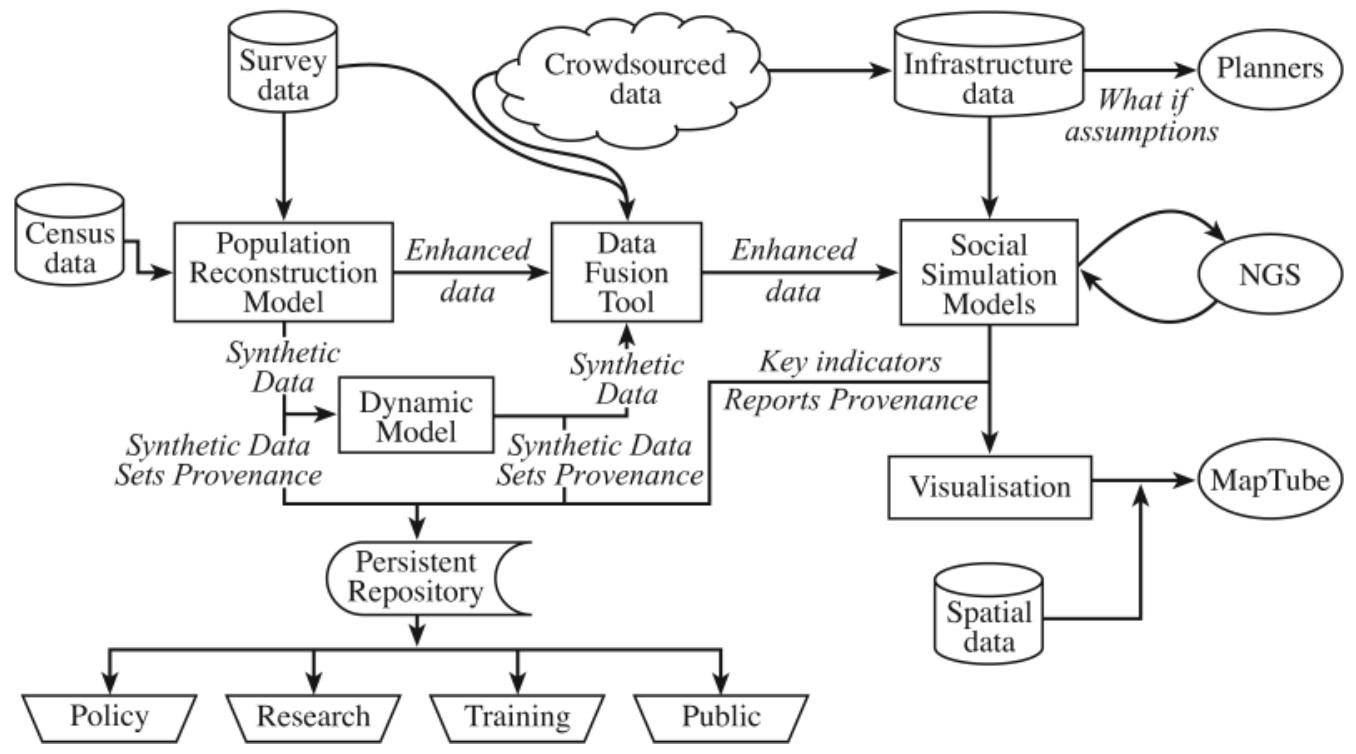

The NeISS architecture depends on e-infrastructure concepts in a number of important ways which are worthy of further brief comment and distinguish the NeISS from a conventional GIS or spatial decision support system. First, the core datasets may be accessed remotely as virtual data stores. In the current implementation of NeISS the data are maintained by the National eScience Centre at the University of Glasgow and accessed through a shibboleth-enabled authentication mechanism through which the regulation of access to secure datasets can be maintained (Shibboleth and UK Access Management Federation, 2010). The simulation engine, 
including both the PRM and DSM, is maintained on a distinct web-server at the University of Leeds. The server is linked to the White Rose Grid node of the UK National Grid Service (NGS: Geddes, 2006) in order to support computationally intensive simulations such as that to be described in Section 3.3 below. The data, simulation models and visualisations are combined as web services into a series of social simulation workflows using the Taverna workbench (Birkin et al, 2010), and these workflows are in turn reconfigured and integrated in order to present portal-based applications which are readily accessed by academic or third-party users (Townend et al, 2009). Specific workflows, and the simulations or policy applications which invoke them, may be archived through a repository management tool known as MyExperiment, where the philosophy is to bundle together specified instances of data, models and visualisation services as publishable Research Objects which may be shared, annotated and re-used (de Roure, Goble, Stevens, 2007). This sharing can take place over popular social networks such as Facebook and Twitter.

Perhaps the most important benefit of the NeISS for spatial analysis and social simulation is that it provides a distributed mechanism for both service delivery and system development. Whilst at present the constituent NeISS services are all developed and delivered by a core team, there is no reason why infrastructure components might not be contributed by a variety of collaborators. The existing team is dispersed widely across the UK, from Southampton and London in the south, to Leeds and Manchester in the north of England, Glasgow and Stirling in Scotland. The scope for adding in new data sources and simulation components within a portalbased plug-and-play workflow architecture is extensive, while if more intensive computation is a requirement then the data and services can be moved easily to match its availability within the network.

\section{Transport simulation within the NeISS}

\subsection{Background}

Following the success of the London Congestion Charge after its introduction in 2005, similar proposals were put forward for other cities, including Manchester. The proposal includes two rings of charging points, the first an inner ring with a radius of approximately 2 miles around the city centre, and the second an outer ring bounded by the M60 motorway. Various pricing options and alternative configurations of the congestion charge zones were offered for public consultation. 
Figure 2. Online Survey for the Manchester Congestion Charge
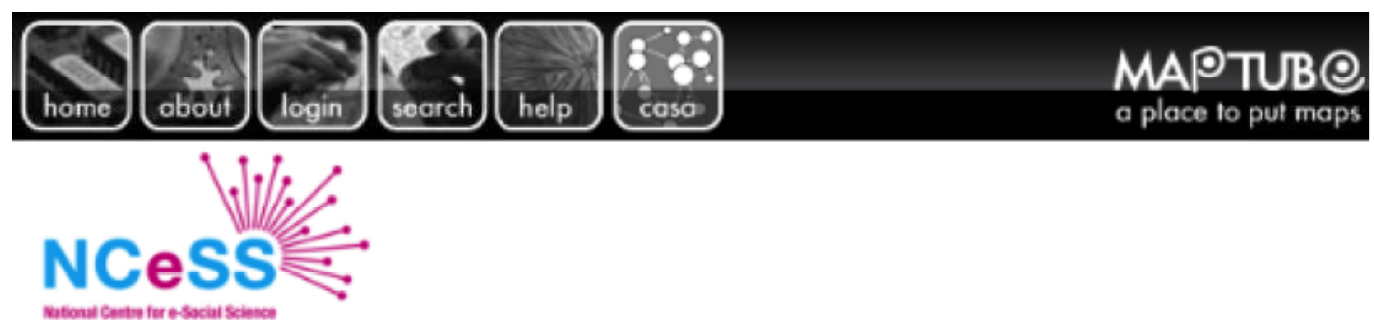

\section{Greater Manchester Congestion Charge \\ Transport proposals: how will it affect you? \\ If you have an interest in how the $£ 2.7$ billion plan to reshape Manchester's transport system will affect your neighbourhood then here's your chance to add what you think to an interactive map of the region. This online collaboration between BBC Manchester and experts at the University of Manchester will give a unique picture of how well the proposals are going down across the north west.}

Please note: the map is refreshed every 24 hours - so you won't be able to see the effect of your selection on your postcode area until the next day.

Simply select one of the options listed below, enter your postcode and click on the submit button. If you just want to see the results, click here: [View Map]

MapTube will include your answer in the next new map.

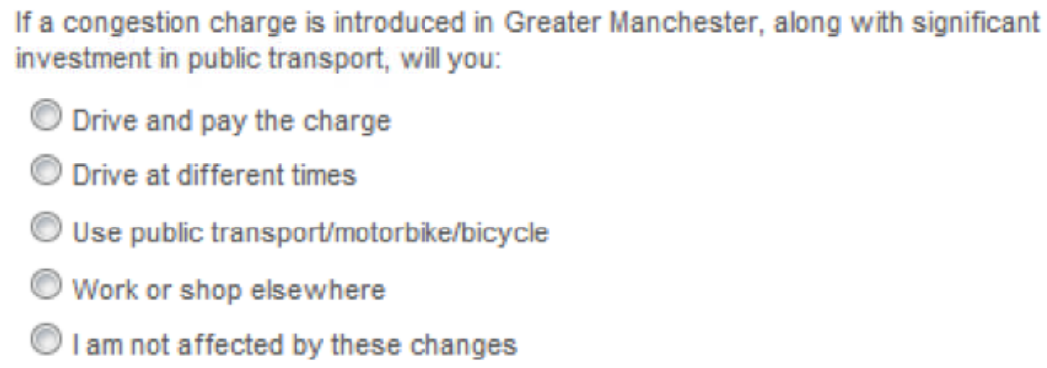

This is NOT a scientific survey or poll and is not connected in any way to the official referendum process.

In parallel to the consultation process a survey was commissioned by BBC North-West from the Centre for Advanced Spatial Analysis (CASA) at University College London in September 2008. Using a simple form connected to the MapTube visualisation software, respondents were 
invited to answer a single question regarding their attitude to the Manchester Congestion Charge (see Figure 2). A residential postcode was requested for each respondent. Following promotion of the survey on local TV and radio, a total of 15,902 responses were received. Views were categorised under 5 headings - (1) would be affected but would not change behaviour, (2) would use a new route or time of travel, (3) would change their method of travel, (4) would select a different destination because of the charge, or (5) not affected by the charge. The results are summarised in Figure 3. The spatial distribution of responses are well spread, with views being expressed from as far away as Aberdeen and Bournemouth. A small number of completed forms amounting to just over 5\% (N=760) were unusable due to invalid or incomplete responses, the most common problem being provision of a postcode which could not be matched to the current version of the Postcode Address File. A notable aspect and risk of this type of crowd sourcing is the possibility of survey manipulation via multiple submissions. We identified two attempts at mass voting, which were picked up by identifying non unique IP addresses and mapping according to response densities.

Respondents were not asked to provide personal details such as age or occupation, however from the postcodes it was possible to undertake geodemographic profiling using the publicly available Output Area Classification of small geographic areas (Vickers and Rees, 2007). The main skew revealed by this analysis was a strong bias towards residents in areas of City Living, and away from Blue Collar Communities ${ }^{1}$. Given that the former are most likely young and prosperous, and the latter much likely to be elderly or deprived this is not entirely surprising.

Figure 3. Results of the Greater Manchester Congestion Charge Survey

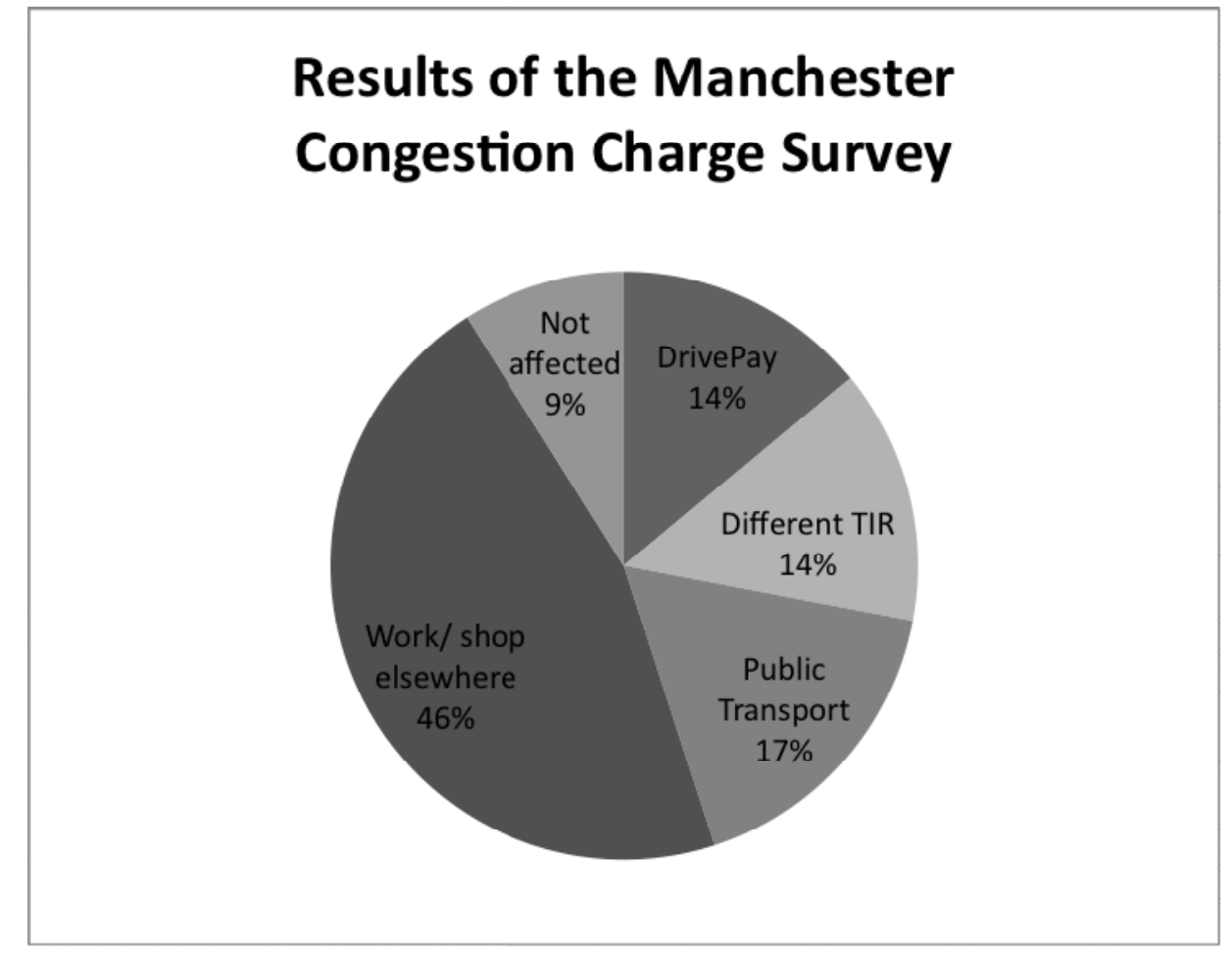

\footnotetext{
${ }^{1}$ The penetration rates for all seven OAC Groups were: Blue Collar Communities, 69; City Living, 171; Countryside, 107; Prospering Suburbs, 102; Constrained by Circumstances, 104; Typical Traits, 107; Multicultural, 108. Penetration of 200 indicates that members of this group are twice as likely to complete the survey; while 50 shows that they are half as likely.
} 
The distribution of responses was used to define an appropriate study region. As we shall see below, the scope of the simulation task bears an exponential relationship to the size of the area, hence limiting the size of this region is of considerable importance. The boundary which was selected is shown in Figure 4, and includes 10,513 or 76.7\% of valid responses to the congestion charge survey. Each of the constituent local authorities shown in Figure 4 was disaggregated into a series of electoral wards, providing a convenient geography for subsequent analysis. There are 214 electoral wards in the extended Manchester region as defined in this way. The figure shows the response rate as the total number of valid responses divided by the number of households in each ward.

From the 2001 Census, this region comprises 2,591,550 individuals spread across 1,086,001 private households. Individuals living outside private households are not considered further in this case study application. The PRM is used to construct a synthetic population in which the household characteristics include location, household size, and housing type, and the individual characteristics include age, gender, marital status, ethnicity, health and occupation. It uses an iterative proportional fitting procedure first introduced to the literature by Birkin and Clarke (1988) and which has been reviewed against alternative techniques with satisfactory results (Harland et al, 2010). A 'complete' set of more than 2.5 million synthetic individuals is generated by the PRM.

Figure 4. Survey Responses and Study Area Definition

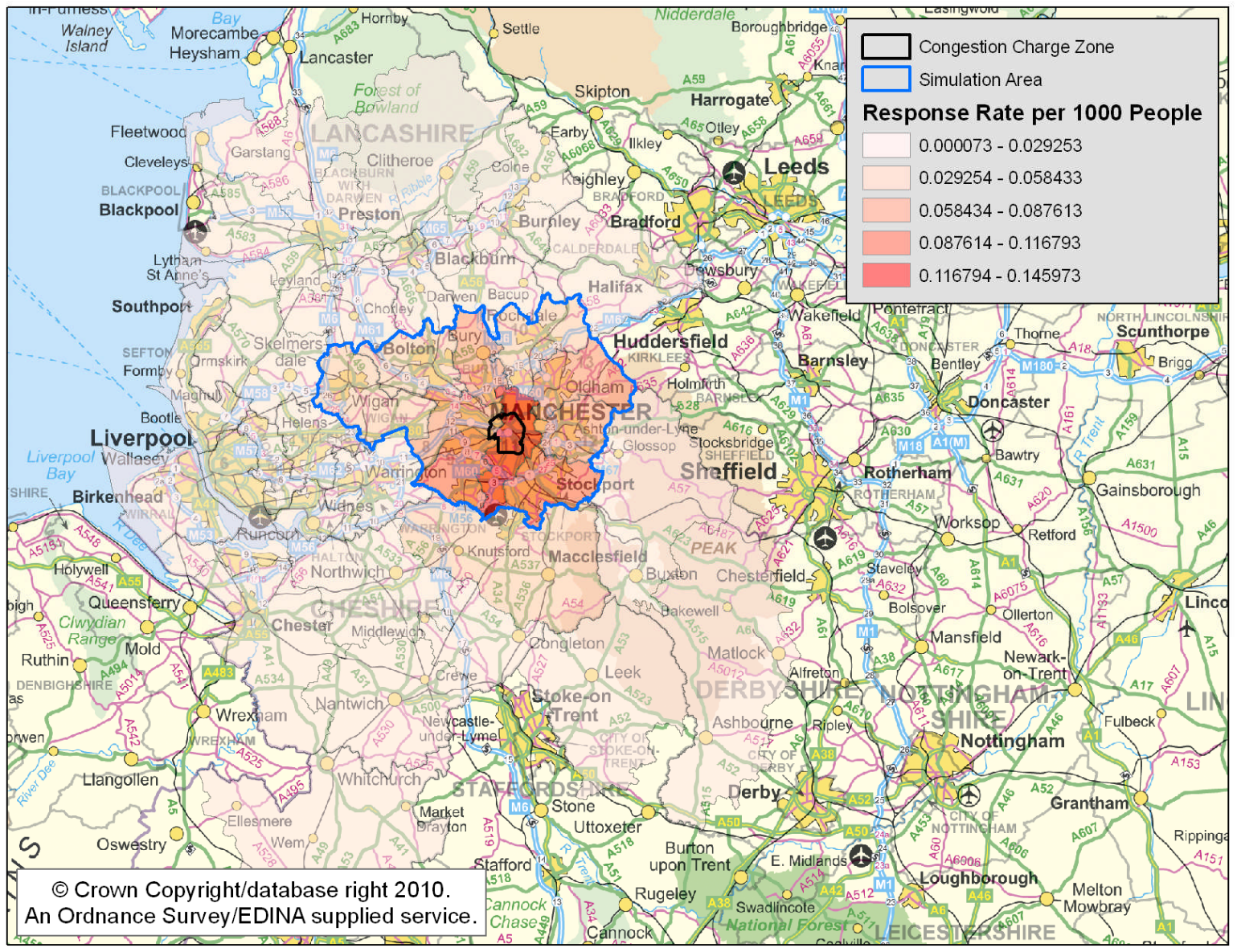

The DSM provides a means for updating and projection of the base population. For the current application, an update from the 2001 Census to the survey date (2008) is required. 
Development work on the DSM allows the evolution of the population to be represented as a series of dynamic transitions including ageing, household formation, migration, fertility, changing health status and mortality (see $\mathrm{Wu}$ et al, 2010). However the estimation of parameters and ground-truthing of the model is an intensive process which has not yet been completed for the areas in this case study. Hence a much simpler proportional updating procedure was adopted. For each local authority area a revised population estimate was obtained from the Office for National Statistics and converted to an appropriate growth rate $g_{i}$. In cases where $g_{i}$ is greater than 1 then households are 'cloned' with probability $\left(g_{i}-1\right)$. In cases where $g_{i}$ is less than 1 then households are eliminated with probability $\left(1-g_{i}\right)$. According to ONS, average population growth of $4.4 \%$ was experienced across the region from 2001 to 2008 .

\subsection{Transport simulation}

The essence of the simulation is to add attributes for each individual, representing a trip destination and the mode of transport associated with this trip. The major part of this task is achieved through a trip assignment model which estimates the number of trips between each pair of zones ( $\mathrm{i}$ and $\mathrm{j}$ ) by mode $(\mathrm{k})$ :

$T_{i j}^{k}=A_{i} r_{i} O_{i}\left(F_{j}^{1}+F_{j}^{2}\right)^{\alpha} \exp \left(-\beta^{k} c_{i j}^{k}+\lambda^{k}+\mu d_{i j}\right)$

$A_{i}=1 / \sum_{j}\left(F_{j}^{1}+F_{j}^{2}\right)^{\alpha} \exp \left(-\beta^{k} c_{i j}^{k}+\lambda^{k}+\mu d_{i j}\right)$

in which $O_{i}$ is the population of zone $\mathrm{i}$, and $r_{i}$ is the average number of trips per year for residents at $\mathrm{i} ; d_{i j}$ is the distance from $\mathrm{i}$ to $\mathrm{j}$ and $c_{i j}^{k}$ is the cost of travelling from $\mathrm{i}$ to $\mathrm{j} . \quad \alpha, \beta^{\mathrm{k}}, \lambda^{\mathrm{k}}$ and $\mu$ are parameters to be estimated in the model.

The attractiveness component of the model represents the two major activities of journey-towork $\left(F_{j}^{1}\right)$ and retail trips $\left(F_{j}^{2}\right)$. Initially we set retail attractiveness to be zero and employment totals for each destination are derived from the 2001 Census. These are then adjusted to maintain the balance between jobs and residents, implying that activity rates are held constant between 2001 and 2008. We estimate attractiveness values for each zone iteratively, so that the sum of the trips into each zone balances to the total number of employment trips at each destination.

Retail attractiveness is generated from an assessment of the size and quality of each retail destination in the region according to Management Horizons ( $M H, 2008)$, whose centre rankings are considered as an authoritative guide by retail analysts. MH identify more than 300 centres in the region, of which flows are modelled to centres ranging in size from Manchester (index $=655$ ) to Wythenshawe (index=51). We use an estimate from the National Travel Survey, which indicates that for every 10 work trips there are 11 associated with retail in order to scale the two attractiveness measures. In other words, we estimate a scalar $\omega$ such that: 
$F_{j}^{2}=\omega M_{j}$

and

$\sum_{j} F_{j}^{2}=\sum_{j} F_{j}^{1} * 1.1$

where $M_{j}$ is the $\mathrm{MH}$ index for centre $\mathrm{j}$.

The trip costs between origin-destination pairs can often be estimated in models of this type using drive times or even straight-line distance. In this case, however, the individual links are extremely important as the levy of a congestion charge is not determined by the origin and destination points but whether the route between them passes through the congestion charge zone. For each i-j pair we generate a set of links so that:

$c_{i j}=\sum_{m n}\left\{\gamma_{i j}^{m} s^{m}+\delta_{i j}^{m n} \theta^{n}\right\}$

where $\gamma_{i j}^{m}=1$ if link $\mathrm{m}$ is on the shortest route from i to j, $s^{m}$ is the length of link m, and $\delta_{i j}^{m n}=1$ if link $\mathrm{m}$ forms part of a congestion charging zone $\mathrm{n}$ with charge $\theta^{n}$ (and zero otherwise, including all cases in which $\mathrm{k} \neq 1$, so the charge applies only to private vehicles). For each link origin-destination pair a route is constructed to minimise the interaction cost in equation (5), from which it can be seen that the congestion charge may leave the link unaffected; the cost of the trip may increase but the route may stay the same; or the route may change as the cost also increases.

Then in the microsimulation, we allocate a trip destination and a transport mode for each of the households in the synthetic population using a Monte Carlo sampling procedure. Each destination and mode is also associated with a route (combination of links $\left.\gamma_{i j}^{m}\right)$ and a $\operatorname{cost}\left(c_{i j}^{k}\right)$.

\subsection{Calibration of the Simulation Parameters}

\subsubsection{Configuration of the Simulation}

The next step in the process is to generate a set of synthetic behaviours within the model described in Section 3.2 which can be aligned with the BBC North-West responses. For the baseline, and for any congestion charge scenario, each individual is attached a destination, mode of transport, trip cost and travel route, using the model described in Section 3.2. If there is no change in any of these attributes between the baseline and the scenario then this individual is assigned to the category 'Response (5) - Unaffected' (see Section 3.1 above, Figure 2 and discussion). The following rules are then applied hierarchically in order to determine the response category for synthetic individuals:

- If there is a change of destination, 'Response (4) - Change of destination'

- If there is a change of route, 'Response (2) - Change of route'

- If there is a change of mode - 'Response (3) - Change of mode'

- If there is a change in the cost, 'Response (1) - Drive and pay'

This assignment process is illustrated in the flow diagram Figure 5. 
Figure 5. Simulated Responses to the Congestion Charge

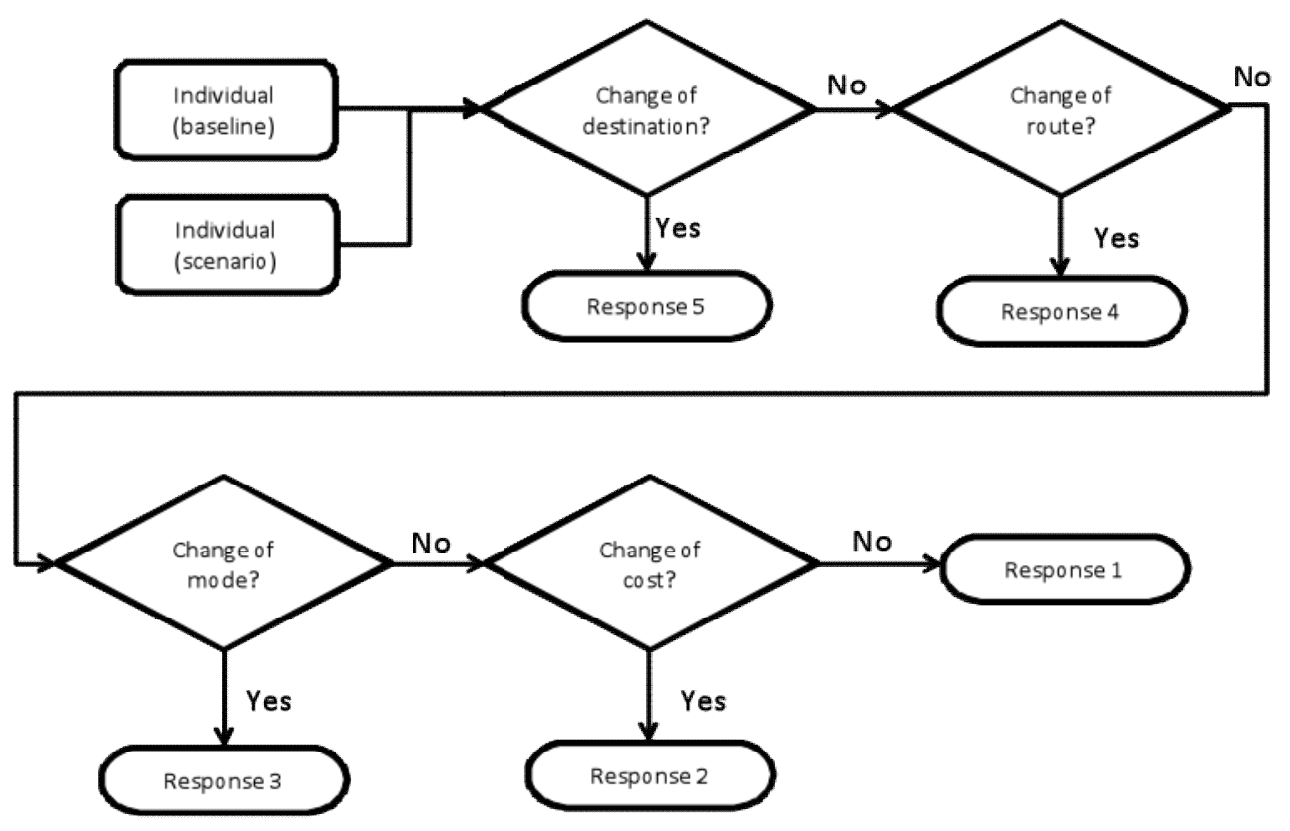

The allocations in both the baseline and the scenario are dependent on the parameter settings for $\beta^{1}$, the distance deterrence associated with trips by car; $\beta^{2}$ the distance deterrence associated with public transport; $\lambda$, the importance of money costs; $\mu$, the importance of nonmoney costs (especially time); and $\alpha$, the attractiveness of Manchester city centre as a retail and workplace destination. However each of the parameters is associated with multiple response variables, and vice versa. For example, if a high value attaches to money costs $(\lambda>>0)$ then the probability of changing destinations will be high, but so will the probability of switching modes and routes. The calibration problem is therefore to find a set of parameter values in which the simulation matches the behaviour of the respondents in the survey data. Because the calibration is complex and non-linear, the problem was addressed using a Genetic Algorithm which is described next.

\subsubsection{Calibration with a Genetic Algorithm}

A genetic algorithm (GA) is a heuristic search routine which takes its inspiration from the concepts of natural selection and survival in the biological world. The algorithm can be used to search for an optimal model configuration in a complex parameter space and is particularly useful for complex problems which require the optimisation of several variables simultaneously (Heppenstall et al., 2007). A GA is based on Charles Darwin's theory of natural selection, whereby small variations in organisms can accumulate if they induce an increase in the overall fitness which improves the individuals' ability to reproduce (Reeves and Rowe, 2003).

The algorithm operates by maintaining a population of individual model parameter configurations (termed 'chromosomes') which, at each iteration, can be combined to form new populations. A chromosome is made up of a number of 'genes' (the five model parameters that 
must be configured for values of $\beta^{1}, \beta^{2}, \mu, \alpha$, and $\lambda$ - see discussion above, Section 3.3.1) and it is the combination of these genes that characterise an individual. Only the most optimal ('fittest') chromosomes remain in the population and form part of the new generation so the overall fitness of the population increases gradually over time. In this manner, the algorithm should converge on an optimal or near-optimal solution. To broaden the search space and prevent the algorithm becoming stuck in non-optimal local minima, mutations can be applied to the new generation whereby the genes in a chromosome are perturbed by a random amount. Figure 6 illustrates the general form of a GA and the remainder of the section will outline some of the individual functions in more detail. The code used in the implementation of the GA is described further in the Appendix (Introduction to the Supplementary Materials) in which instructions are also provided for users wishing to download the software and data for the purposes of their own research.

Figure 6. A Genetic Algorithm for Parameter Selection

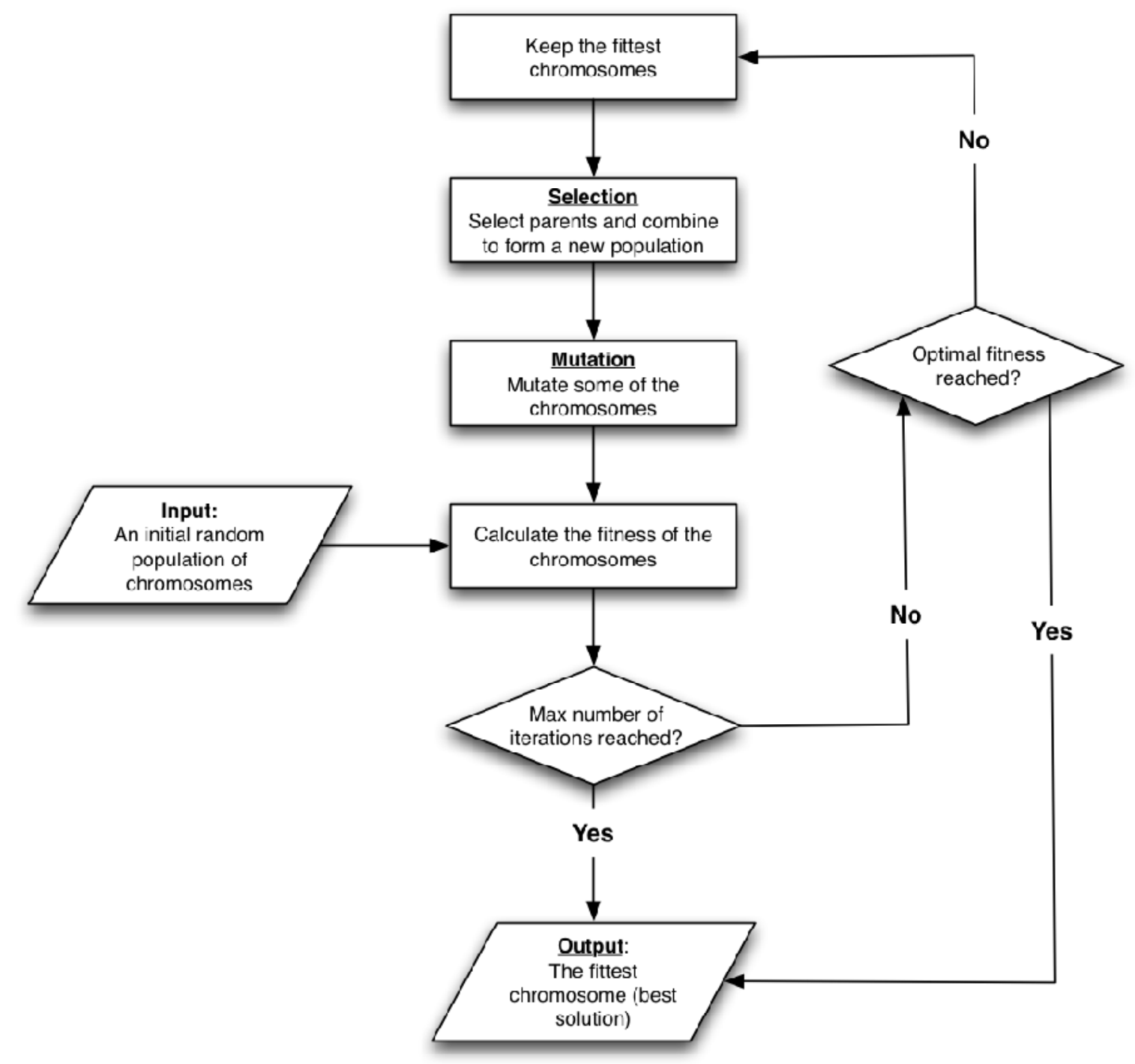




\section{$\underline{3.3 .3 \quad \text { Fitness }}$}

Using the rules expressed in Figure 5 above, model-based estimates were generated for each of the behaviours which were captured in the congestion charge survey ${ }^{2}$. Labelling each of these stated behaviours as B(n), and the associated model estimate as B" $(n)$ then the goodness of fit of the model is evaluated as the sum of differences between the estimates and the observations:

$G=\sum_{n=1}^{5}\left|B(n)-B^{\prime \prime}(n)\right|$

One of the most important difficulties in the modelling is the very high levels of impact reported from the Congestion Charge Survey. For example, nearly half of responders stated that they would 'work or shop elsewhere', while in practice it seems unlikely that as many people living in the study area will work or shop in the centre of Manchester to begin with. In order to resolve this difficulty, data on the distribution of trips from the National Travel Survey was used, showing that nearly half (49\%) of trips are for purposes other than work and shopping (i.e. education or leisure). Since these 'other' trips are mostly local they are much less likely to be affected by the congestion charge boundary. We assume the responses to the question 'not affected by the charge' relates only to non-local trips, which we adjust up to $54 \%(0.49+$ $0.09 * 0.51$ ) and the other responses were down weighted proportionately. The calibration was optimised to the following proportions:

$\begin{array}{lr}\text { B(1) - Carry on } & 7 \% \\ \text { B(2) - Change route } & 9 \% \\ \text { B(3) - Change mode } & 7 \% \\ \text { B(4) - Change destination } & 23 \% \\ \text { B(5) - Unaffected } & 54 \%\end{array}$

\section{$\underline{3.3 .4 \quad \text { Selection }}$}

Selecting parents based on their fitness is the means by which the GA converges on an optimal solution. In this implementation, roulette wheel selection is used such that the probability of a chromosome being selected to form the next generation is proportional to its share of the total fitness of all chromosomes in the population. However, so that the maximum fitness of the population never decreases, the fittest chromosome is always selected (Reeves and Rowe, 2003).

\subsubsection{Breeding a new generation}

There are numerous methods for combining parents to form children chromosomes and in this implementation intermediate recombination is used. With this method, values for each of the child genes are chosen from within the range of the parent's genes. To retain diversity, this range is extended by $+/-25 \%$ ensuring that the gene space of new children does not decrease with time.

\footnotetext{
${ }^{2}$ For the purpose of estimating the models in this paper, it has been assumed that stated preferences are a reliable indicator of revealed behaviours. Although this simplifies our analysis, the integrity of the model is not seriously compromised by this assumption.
} 


\section{$\underline{\text { 3.3.6 Mutation }}$}

To make sure that a large search space is covered, it is essential that a GA retain diversity (Holland, 1975) and mutation is a means of both increasing diversity and also exploring existing areas of the search space. The principle of mutation is that a gene (or a number of genes) have their values changed by a chosen amount. This amount can decrease over time so that the algorithm can narrow down to optimal solutions.

\subsection{Implementation on a computer grid resource}

Calculating the fitness of a chromosome requires running an instance of the transport model with the given parameter/gene configuration. Over the course of a GA execution, large numbers of individual model runs must therefore be conducted (the size of the population of chromosomes multiplied by the maximum number of GA iterations). As these individual models do not interact with each other, the situation is ideally suited for 'lazy parallelization'. This is a method of parallelization whereby a single compute node can execute a model without any intervening communication with other nodes; reducing communication overheads and requiring minimal changes to an existing non-parallel model.

To run the model on a grid, access to the National Grid Service (NGS) compute resources were provided and the GA algorithm has been implemented in Java using the MPJExpress messagepassing interface. A 'master' node is responsible for maintaining and evolving the population of chromosomes, but the task of calculating the fitness of a chromosome is delegated to slave nodes. In this manner, a GA run of 100 iterations and a population of 100 chromosomes can be executed in under 10 minutes using 101 separate compute nodes (100 slaves and one master). Running the equivalent algorithm on a single machine could take over 13 hours (approximately 4.8 seconds per model, $100 * 100$ individual model runs).

\subsection{GA Results}

Initially, parameter values are set at random between 0 and 10 for the five parameters discussed in Section 3.3.1 $\left(\beta^{1}, \beta^{2}, \lambda\right.$, $\mu$, and $\left.\alpha\right)$. The performance of the algorithm is illustrated in Figure 7, which plots the magnitude of the error between the transport model results and the expected data (computed according to equation (6)). Only the model with the best fit (lowest error) is shown for each iteration. Over the course of the calibration, a substantial improvement in the model fitness is evident. Whilst progress is initially rapid, better solutions continue to be found, even after relatively long periods of stability. For example, there is no change between iterations 40 and 75, but then a small change is quickly followed by more jumps in the quality of the solution. After 110 iterations the lowest error was 0.141 , and we found that extending this further to 500 iterations yielded no further improvements in the model goodness-of-fit. 
Figure 7. Fitness of the GA Calibration

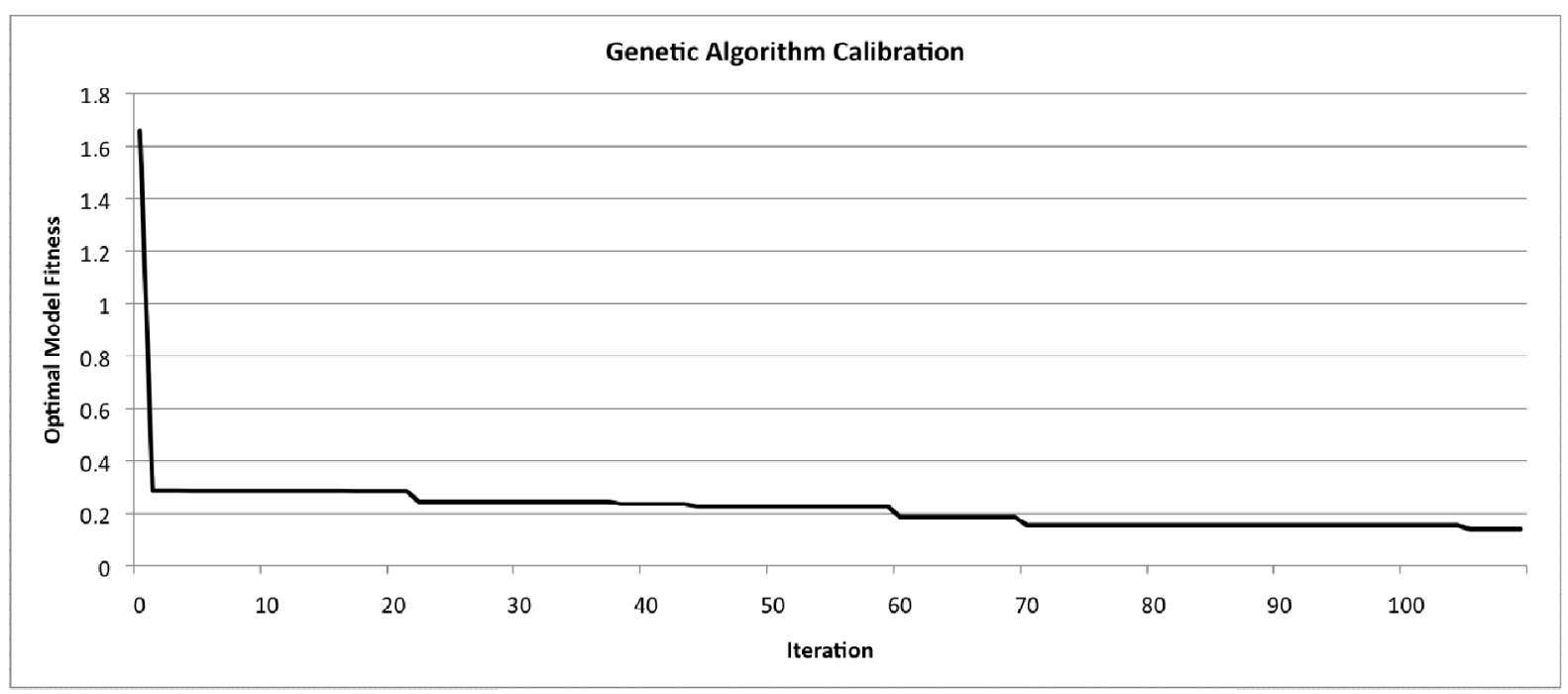

The best fit parameter values from the model optimisation are shown in Table 1. For the remainder of this section, this configuration of values will be used to explore scenarios in which travel patterns are subject to change under the influence of a congestion charge.

Table 1. Gene values of the optimal model following GA calibration.

\begin{tabular}{|l|l|l|r|}
\hline Gene & Parameter & Description & Value \\
\hline 0 & $\beta^{1}$ & distance deterrence - trips by car & 0.38 \\
\hline 1 & $\beta^{2}$ & distance deterrence - public transport & 0.53 \\
\hline 2 & $\lambda$ & the importance of money costs & 1.09 \\
\hline 3 & $\alpha$ & $\begin{array}{l}\text { the attractiveness of Manchester city } \\
\text { centre }\end{array}$ & 1.08 \\
\hline 4 & $\mu$ & the importance of non-money costs & 0. \\
\hline
\end{tabular}

\subsection{Model outcomes}

The key behavioural outcomes of the survey are reproduced in Table 2, along with the equivalent model predictions in which 33 wards are selected to approximate the congestion charging area shown in Figure 4, and in which the congestion charge is set to $£ 5$. This configuration is referred to as Scenario 1, and generates model behaviours which are closely aligned to the survey results. The model is then run for a second time with the same area, but with the charge raised to $£ 10$ (Scenario 2). In this case, some significant changes of behaviour can be seen. In particular, the number of people willing to carry on regardless of the charge is reduced significantly, and instead people are much more likely to find a new destination or to travel by a different mode.

Table 2 also shows results from scenarios in which the charging area has been approximately doubled in size to include 69 wards in the Manchester area. This is shown for Scenario 3, with a charge of $£ 5$, and for Scenario 4 with a charge of $£ 10$. Again the most noticeable effects are a big increase in switching both modes and destinations, while the number of people affected by the charge is successively reduced. Through simulations of this type, planners could therefore begin to evaluate changes in travel behaviour from the implementation of different policy alternatives using evidence which has been generated as volunteered geographical information. 
The software and data which were used in these simulations is described further in the Appendix (Introduction to the Supplementary Materials) in which instructions are also provided for users wishing to download these files for the purposes of their own research.

Table 2. Travel behaviour in the Survey and in the Congestion Charge Scenarios

\begin{tabular}{|l|l|l|l|l|l|}
\hline & Survey & Scenario 1 & Scenario 2 & Scenario 3 & Scenario 4 \\
\hline & & $\begin{array}{l}33 \text { wards in } \\
\text { central ring } \\
\text { with f5 charge }\end{array}$ & $\begin{array}{l}33 \text { wards in } \\
\text { central ring } \\
\text { with f10 } \\
\text { charge }\end{array}$ & $\begin{array}{l}69 \text { wards in } \\
\text { extended ring } \\
\text { with f5 charge }\end{array}$ & $\begin{array}{l}\text { 69 wards in } \\
\text { extended ring } \\
\text { with f5 charge }\end{array}$ \\
\hline Carry On & $7 \%$ & $9 \%$ & $2 \%$ & $10 \%$ & $2 \%$ \\
\hline Change Route & $9 \%$ & $6 \%$ & $4 \%$ & $5 \%$ & $2 \%$ \\
\hline Change Mode & $7 \%$ & $7 \%$ & $12 \%$ & $12 \%$ & $22 \%$ \\
\hline $\begin{array}{l}\text { Change } \\
\text { Destination }\end{array}$ & $23 \%$ & $23 \%$ & $27 \%$ & $24 \%$ & $30 \%$ \\
\hline Unaffected & $54 \%$ & $55 \%$ & $55 \%$ & $49 \%$ & $44 \%$ \\
\hline
\end{tabular}




\section{Discussion}

There are a number of reasons to treat the specific results from the simulation exercise with caution. Regarding the survey itself, the number of people who felt they were unlikely to be affected by the charge is surprisingly low, especially in view of the fact that many would be expected to travel by public transport and others will tend to live, work and shop in freestanding satellite towns which would be largely unaffected. It seems likely that the survey has attracted a skewed response from those who have particular concerns about this policy. There is also uncertainty in both the model and the survey itself about the nature and extent of the charge. At the time the survey was undertaken, the size of the charge, its structure (for example, a high rate central charge and a lower rate for the periphery), and the physical extent of its coverage were still open for debate and consultation. Within the model, although it was possible to explore a number of alternative scenarios, the calibration is derived from a fairly straightforward representation of the congestion charge area and one which is unlikely to match exactly either the perception of our survey respondents or the final implementation of the policy.

The model itself is also affected by a number of error sources. The size of the study area has been restricted, partly to keep the matrices of links connecting each pair of origin and destination nodes to a manageable size. Although the study area accounts for nearly $80 \%$ of respondents to the MapTube survey, it is clear that there are many people outside this area who have strong views and are likely to be affected. The interactions with large surrounding conurbations such as Liverpool and Leeds cannot be allowed for without a significant increase in the range of the model. In relation to the calibration itself, we chose to utilise single regionwide parameters rather than trying to represent spatial or social variations in behaviour and attitudes, for example that older and more affluent people are perhaps more likely to have a positive view of car ownership and use. From the inspection of the GA results it seems likely that the model optimisation is far from monotonic, and that particularly with uncertain data inputs a marginal variation in the assumptions or response behaviours could give rise to a rather different model parametrisation, from which inconsistent policy conclusions might be drawn.

Many of these problems could be addressed by drawing more well-established simulation technologies into the NeISS infrastructure. There are software packages in abundance for the estimation of travel patterns in both the academic and planning domains (e.g. Paramics, Transim, Saturn, Omintrans and many others). By designing an open, service-based infrastructure there is no reason why such third party software might not be incorporated as plug-and-play services within the infrastructure, although major issues of licensing and copyright would need to be overcome in order to achieve this. One possible solution here would be to configure the e-infrastructure into specific decision-support applications, so that if for example the Greater Manchester Transport Executive is an established user of Paramics, then this can be configured as the simulation engine within a congestion charge study for Manchester. An example of such a decision-support system is described by Townend et al (2009), and its architecture including the necessary security requirements is discussed further in Birkin et al (2010). 
Notwithstanding these reservations, the capability to link crowd-sourced data, visual simulation, scenario evaluation and analysis within the e-infrastructure which has been proposed in this paper provides a valuable framework for both policy-making and the academic study of urban and regional systems. Since the survey of attitudes to congestion charging which have been reported above, further developments in the MapTube software have facilitated the development of a discrete SurveyMapper toolkit. After a straightforward registration procedure, SurveyMapper allows users to generate questionnaires, to post these online, and map the results in real time. The package is accessible to computationally unsophisticated users (for more discussion, results and analysis, see Hudson-Smith et al, 2010). While the distribution of online surveys remains a significant obstacle, and the response biases will be heavily skewed by the digital divide (Longley and Singleton, 2009), nevertheless crowd-sourced data generation is clearly now cheap, workable and egalitarian. It would be surprising to see anything less than a dramatic upswing in data gathering through these methods in both academic and commercial applications such as market research (Savage and Burrows, 2007).

This paper specifically raises the possibility of crowd-sourced data as a mechanism for the calibration of policy models. Secondary data from sources like the 2001 Census and National Travel Survey will continue to provide a robust basis in fact for model-based planning. However the inclusion of response data which is both up-to-date and specific to the problem at hand is potentially an appealing possibility. An aspect of this which we intend to pursue in future work is the addition of a further feedback loop from the simulation back to the crowd. Thus having given their opinions on the effect of a policy on their behaviour, responders (e.g. the general public) might then explore the results of simulations, using a platform such as NeISS to gauge the effects of their behaviour when extrapolated back to the real world. Following the MapTube survey in September 2008 the Manchester Congestion Charge was rejected overwhelmingly by a local public vote. Apparently the population were influenced by the immediate financial effects and inconvenience of the scheme. Whether public opinion might have been swayed by the provision of simulations showing the effect of their decisions on future congestion patterns, economic activity or other indicators is debatable. However a strong case can be made that this would add a valuable perspective to the planning process.

\section{Conclusions and Next Steps}

In this paper a straightforward case study of a traffic simulation for the Manchester congestion charge has been used to illustrate a portal-based simulation architecture for spatial planning. This illustration has been based on a public consultation commissioned by an influential media group (the BBC). The next stage in the development is to develop use cases in relation to real planning situations. The NeISS team is currently working with a European project examining urban population dynamics, alongside an academic study of the impact of family background and social circumstances on education and life chances, with a group of users to articulate potential social policy applications in educational attainment, life chances and inequality, and with health care planners exploring scenarios for resource utilisation, need and service provision.

It has been argued that Web 3.0 tools, such as SurveyMapper, provide a powerful means for the engagement of relatively unsophisticated users within the analytical simulation process. At the 
same time, the possibly of including more powerful algorithms e.g. for traffic simulations has been noted. The project will continue to cater for a multi-layered community, ranging from the naive (contributors of data and potentially consumers of simulation outputs), through competent users (who might wish to employ reasonably complex models, analytics and visualisation tools), on to development partners, who even have the capability to contribute additional tools to the technologies already available.

\section{Acknowledgements}

The research reported in this paper has been funded through the JISC Information Environments Programme, e-Infrastructure for Social Simulation.

\section{References}

Anand, S., Batty, M., Crooks, A., Hudson-Smith, A., Jackson, M., Milton, R., Morley, J., and Rosser, J. (2010) Data Mash-Ups and the Future of Mapping, JISC TechWatch, Technology and Standards Watch, Joint Information Systems Committee, Bristol, UK.

Bell, G., Hey, T. and Szalay, A., 2009. Beyond the Data Deluge, Science, 323 (5919), pp. 12971298.

Benenson, I. and Torrens, P., 2004. Geosimulation: automata-based modeling of urban phenomena. Wiley.

Birkin, M. and Clarke, M., 1988. SYNTHESIS: A SYNTHetic Spatial Information System for urban modelling and spatial planning. Environment and Planning A, 20, 1645-1671.

Birkin, M., Townend, P., Turner, A., Wu, B. and Xu, J., 2009. MoSeS: A Grid-enabled spatial decision support system, Social Science Computer Review, 27, 493-508.

Birkin, M., Procter, R., Allan, R., Bechhofer, S., Buchan, I., Goble, C., Hudson-Smith, A., Lambert, P., de Roure, D. and Sinnott, R., 2010. The Elements of a Computational Infrastructure for Social Simulation. Philosophical Transactions of the Royal Society A, 368 (1925), 3797-3812

De Jong, K. A., 2006. Evolutionary Computation: A Unified Approach. MIT Press.

De Roure, D., Goble, C. and Stevens, R., 2007. Designing the myExperiment Virtual Research Environment for the Social Sharing of Workflows. Proceedings of the Third IEEE International Conference on e-Science and Grid Computing, Bangalore, India, December 2007, Washington, D.C.: IEEE Computer Society, pp. 603-10.

Epstein, J., 2007. Generative Social Science: Studies in Agent-based Computational Modelling. Princeton, NJ: Princeton University Press.

Eubank, S., Guclu, H., Anil Kumar, V., Marathe, M., Srinivasan, A., Toroczkai, Z., Wang, N., 2004. Modelling disease outbreaks in realistic urban social networks. Nature, 429 (6988), 180-184. 
Geddes, N., 2006. The National Grid Service of the UK. Paper presented at the International Conference on e-Science and Grid Computing, Los Alamitos, CA, USA.

Geertman, S. and Stillwell, J., eds, 2003. Planning support systems. Berlin: Springer.

Goodchild, M.F. (2007) Citizens as Sensors: The World of Volunteered Geography. GeoJournal, 69 (4), pp.211-221.

Harland K., Birkin M., Heppenstall A. and Smith, D., 2010. Creating realistic synthetic populations at varying spatial scales: A comparative critique of microsimulation techniques. Journal of Artificial Societies and Social Simulation, in press.

Heppenstall, A. J., Evans, A. J. and Birkin, M. H., 2007. Genetic algorithm optimisation of a multiagent system for simulating a retail market. Environment and Planning B 34 (6) 1051-1070.

Holland, J. H., 1975. Adaption in Natural and Artificial Systems. University of Michigan Press.

Hudson-Smith, A., Birkin, M., Milton, R., Gray, S. and Neuhaus, F., 2010. Harvesting the Crowd for e-Science, and Simulation: the SurveyMapper toolkit. Paper presented at the e-Science All Hands Meeting, Cardiff.

Longley, P. and Singleton, A., 2009. Linking Social Deprivation and Digital Exclusion in England. Urban Studies, 46 (7), 1275-1298.

MHE Retail Limited, 2008. Management Horizons Europe: UK Shopping Index.

Miller, E. J., Hunt, J. D., Abraham, J. E. and Salvini, P. A., 2004. Microsimulating Urban Systems. Computers, Environment and Urban Systems, 28 (1), 9-44.

OECD (2007) Participative Web: User-created Content:. Organization for Economic Cooperation and Development, 12th April 2007. Available online as:

http://www.oecd.org/dataoecd/57/14/38393115.pdf

Raney, B. and Nagel, K. , 2004. Iterative route planning for large-scale modular transportation simulations. Future Generation Computer Systems, 20 (7), 1101-1118

Reeves, C. R. and Rowe, J. E, 2003. Genetic Algorithms - Principles and Perspectives. Kluwer Academic Publishers.

Savage M. and Burrows, R., 2007. The Coming Crisis of Empirical Sociology. Sociology, 41, 885899.

Shibboleth and UK Access Management Federation, http://www.ukfederation.org.uk/

Townend, P., Xu, J., Birkin, M., Turner, A. and Wu, B., 2009. Modelling and Simulation for e-Social Science. Philosophical Transactions of the Royal Society A, 367, 2781-2792.

Vickers, D.W. and Rees, P.H. (2007). Creating the National Statistics 2001 Output Area Classification. Journal of the Royal Statistical Society, Series A, 170, 2, 379-403.

Waddell, P. and Borning, A., 2002. A Case Study in Digital Government: Developing and Applying UrbanSim, a System for Simulating Urban Land Use, Transportation, and Environmental Impacts. Social Science Computer Review, 22 (1), 37-51. 
Warner, G. C., Blum, J. M., Jones, S. B., Lambert, P. S., Turner, K. J., Tan, L., Dawson, A. S. F. and Bell, D. N. F., 2010. A social science data-fusion tool and the Data Management through e-Social Science (DAMES) infrastructure. Philosophical Transactions of the Royal Society A, 368 (1925), 3859-3873

Wu, B., Birkin, M. and Rees, P., 2008. A spatial microsimulation model with student agents. Computers Environment and Urban Systems, 32, 440-453.

Wu, B., Birkin, M. and Rees, P., 2010. A dynamic MSM with agent elements for spatial demographic forecasting. Social Science Computer Review, forthcoming

\section{Appendix: Introduction to the Supplementary Materials}

The model code and data which were used in the preparation of this paper are available as a download for readers of the International Journal of Geographical Information Science. These supplementary materials are bundled into a single zipfile, and the content and implementation of the model is described in the associated 'readme.txt' document. Users of the software will be able to recreate the calibration of the simulation parameters using a Genetic Algorithm (GA), which is described in Section 3.3, and to reproduce the experimental results which are discussed in Section 3.6 and presented in Table 2 of the paper.

The GA is implemented in java and can be configured for a range of populations of chromosomes and model iteration cycles. The results reported in the paper were generated with 100 chromosomes over 500 iterations, the congestion charge was set at $£ 5$, and the 'normal' congestion zone was selected (as described in Section 3.6). However the algorithm uses randomly generated seeds so we would expect to see some degree of variation whenever these experiments are reproduced. Lower quality solutions would be expected if the population size or iteration limit is reduced, although this would also speed up the execution time for the algorithm.

The transport simulation model is written in FORTRAN, and uses parameter values derived by the GA. The model can be executed using either the 'normal' or 'extended' congestion charge area, and the level of the charge can also be varied through the user interface. Thus the model results presented in Table 2 of the paper can be recreated with the normal or extended congestion zone, and a charge of either $£ 5$ or $£ 10$. Other outcomes not reported in the paper can be generated by varying the cost of the congestion charge (i.e. values other than $£ 5$ or $£ 10$ ). More ambitious users could consider the creation of other congestion zones by manual editing of the control file (TCONTROL.TXT) rather than automatic generation through the simulation procedure. Alternative parameter settings can also be effected through manual editing of the control file (for example, increasing beta to represent the effect of rising fuel prices). The simulation code also supports other interventions such as road pricing and priority lanes for public transport. 